TABLE III.-Cases known to have Died taken together with those still Alive and Examined as to Duration of Life.

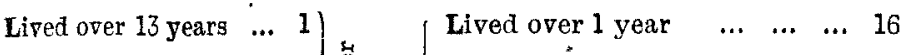

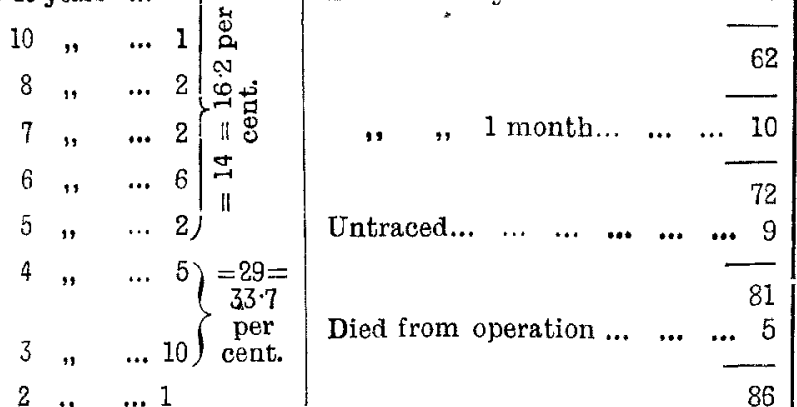

From this it will be seen that 16 per cent. lived over five years and 337 per cent. over three years. These figures appear to justify the hope that the expectancy of life is improving as time goes on. It is clear that over 33 per cent. live more than three years after the operation, which has suggested to some surgeons that such cases should be regarded as permanent cures. But some of the cases (26.9 per cent.) have died most likely from recurrence in all but one after an interval of immunity of over three years, so that such a presumption is unwarranted.

Another interesting point comes out from this analysisnamely, that only seven suffered from local recurrence. Those in whom the disease has returned have shown it in internal parts. This is another good feature of the modern operations, local recurrence being most depressing and painful, while internal generalisation is often not sc.

These cases have been accurately recorded and in almost every case the diagnosis with the naked eye has been confirmed by microscopical examination by various competent observers, mostly the hospital registrars. In the oldest case in the list the glands were extensively invaded as well as the breast, and a bad prognosis was given at the end of the operation, and yet at the end of 14 years the patient is well and free from any trace of cancer. This and some of the other cases seem to justify the hope that this disease can be eradicated by the knife. And it is not too much to hope that with still further improvement in early diagnosis and treatment a far larger proportion of cases may be saved. If the four cases of duct cancer were included the figures would be still more favourable.

\section{ON THE PATHOLOGY AND THERAPY OF ANGINA PECTORIS.}

By Professor THEODOR SCHOTT, M.D.

Ayroxg chronic diseases of the heart angina pectoris bas in the passing century attracted the interest of the medical profession in a remarkable manner. Ever since the first excellent description by Heberden appeared more than 100 years ago the various theories advanced as to the nature and the causes of stenocardiac accesses have been in sharp opposition. This may be explained in great part by the fact that, as Latham justly remarks, angina pectoris must be regarded not as a disease sui generis but as a series of symptoms. Even, however, in regard to angina pectoris vera-to which the following observations are exclusively directed--opinions still remain unsettled and divided. The hypothesis of Heberden and of Latham that the anginal fit is to be regarded as a real tetanic contraction of the cardiac muscle has found at different times a varying number of adherents. A tetanic cramp of the whole cardiac wall would, if it prevailed but a short time, certainly destroy human life; but a tetanic contraction of a part of the heart might take place without deadly result, as has been demonstrated latterly by experiments of Basch and Grossmann. In direct opposition, however, is the theory of Parry which has been effectively supported by the celebrated Stokes. According to this opinion angina pectoris must be attributed, not to an increase but to a further reduction of the muscular energy of a heart already enfeebled. According to Parry the stenocardia is a sort of syncope with a preceding strong oppression or pain in or near the heart, the consequence of an organic lesion the effects of which are brought into evidence by a lowering of heart power. Thus Parry opines that the symptoms are caused by an accumulation of blood in the cardiac cavities. This explanation has been powerfully supported in Germany by the well-known Professor Traube, an authority on the subject of heart diseases. Traube also believes that there is loss of power of the heart muscle, but he thinks that the access results from a rapidly increasing tension of the walls of the ventricles under the influence of an accelerated over-filling of the cardiac cavities, in consequence of which the motor as well as the sensor nerves of the heart respectively are subjected to a degree of pressure or tension sufficient to account for the sudden pain. Samuelson, whose experiments are founded upon an artificial constriction of the coronary vessels, differs from Traube only in that he supposes the overfilling of the heart to take place, not in the left ventricle but in the right auricle. French physicians, such as Potain, Germain Sée, and others, attribute the attack to an ischæmic state of the cardiac muscle, attributing the pains to causes similar to those observed when there is a blocking of the arteries as in endarteritis obliterans and in similar processes. But the ischaemic state of the cardiac muscle may be taken to indnce a weakness of the organ, so that this theory agrees in the main with that of Parry and Stokes. Although the latter view commands a majority of adherents-as may be gathered, for example, from the opinion expressed at the meetings of the London medical societies - it has always found opponents as at the Congress of Internal Medicine at Wiesbaden (1891) where Vierordt, one of those who opened the discussion, rerired the question whether the stenocardiac fit is really dependent on debility of the heart. The principal argument for that doubt is that the pulse does not of necessity show any corresponding change-that there may be no diminution of volume-that in some cases, indeed, it becomes stronger. Another argument is that manifest signs of weakness of the heart are not invariably discoverable.

Before we discuss these theories let us consider what processes are found to be associated with angina pectoris vera. They are, in the first place, sclerosis of the coronary vessels, alterations of the aortic valves, and aortitis, especially that form which causes an ectasia of the ascending portion. (In a case of angina pectoris combined with insufficiency of the mitral valve which has been under my treatment these three years I observed latterly the development of an aortic stenosis; so that also in this case, though there are no signs of rigidity of the vessels, the sclerotic process at the heart cannot be regarded as doubtful. I may add that latterly I have seen several similar cases.) It is known, however, that the sclerotic change in just this part of the aorta is not without an influence on that spot from which the coronary vessels take their origin, and not seldom it may be noted post mortem that, though the other parts of the coronary arteries still show a sufficient lumen it is reduced at the point referred to in such a degree that a bristle can scarcely be introduced. The next consequence of such an arterial change must be that as a result of decreased circulation the muscles of the heart are no longer adequately nourished. Wealsening of the heart and defect of energy follow of necessity. Moreover in the stenocardia of angina pectoris vasomotoria - the pseudo angina resulting from so many different causes may be left aside-the heart is called on to exert itself against a contracted arterial system, so that in such cases, also, there is a sufficient cause for a sudden relative wreakness of the heart. The conditions calculated to induce a weakening of the heart are apparently present in both instances, and it is easy to understand that every demand for additional effort is likely to be followed by an additional, as well as sudden, failure of energy. The suddenness of the paroxysm may be also directly attributable to the retardation of the circulation within the coronary vessels. A moderate distension of the cardiac muscle may easily lead to a temporary ccclusion of one or more coronary vessels at the seat of an already existing constriction. In other cases a thrombus or an embolus are the cause of the block, and we know that such vascular conditions give rise to violent as well as sudden pain in other parts of the muscular system. The experiments of Samuelson, of Cohnheim, of Schultess-Reibberg, and of many others have furnished sufficient evidence of this.

Let us now inquire whether clinical observation lends confirmation to what has been suggested. I restrict myself to my own experience, and $I$ would in the frst place 
emphasise the fact that I never could observe any absolutely characteristic or invariable condition of the circulatory system before, during, or after the accesses. Let us look first to the state of the pulse. For that purpose we must not entirely depend on digital examination-we must have recourse to the sphygmograph and the sphygmomanometer. How often do we recognise by means of these instruments that, for instance, a bradycardia, which actually exists in some instances, is in a number of others only apparent, and that the sphygmograph discloses a number of pulsations which are not appreciable by the finger so that the apparent bradycardia may be an actual tachycardia. In other cases we meet with a real tachycardia or an intermittent or arhythmic pulse. That the volume of the pulse very often appears reduced is well known. With a large number of patients $I$ have noted reduction of the pressure of the pulse during the attacks. The latter observation is especially applicable to the pulsus celer, the tension of which is frequently apt to lead to mistakes.

We will now proceed to the examination of the heart itself. In the earliest stages of this cardiac disease it is frequently very difficult to certify to difference in the strength and character of the heart's sounds in the intervals between the attacks as compared with those of unimpaired health. There are other cases in which the contrast becomes rapidly apparent. It will not, however, escape the attention of a practitioner who has occasion to observe such patients for a long period that heart-sounds become feebler during the fits; in other cases the sounds become dull or impure and distinct murmurs are observable, and not rarely I have convinced myself that the rhythm assumes the character of a gallop. Less frequently the beatings of the heart become fotal or fluttering.

Evidence still more demonstrable may be obtained by percussion. $^{1}$ The limits of dulness have been accurately traced with a solution of nitrate of silver. The following may be observed more or less definitely according to the progress of the disease. In the beginning there is a moderate dilatation which affects especially the left side. For a time the left auricle may-be more distended than the corresponding ventricle. If the malady advances the heart becomes more and more distended; the right side becomes involved, but the left ventricle remains more permanently dilated until finally the whole heart is and remains enlarged. In such cases-that is, those in which the limits of the heart fail to recede to a normal state-we note that with the stenocardia there co-exist the other indications of a cardiac dilatation with consequent incompetence, such as breathlessness, \&c., but the characteristic feature which is attested by percussion at the beginning of the disease is debility of the left heart. It has been a satisfaction to me to be able to confirm these results of percussion by means of the $x$-rays, which supply an important additional resource for physical examination. I had an opportunity last year when I made a number of experiments with Roentgen rays in cases of cardiac muscular diseases, not only to examine with the fluorescent screen but to take a radiogram of a man 40 years of age at the very moment in which he became subject to an anginal fit. This patient had in former years consumed quantities of beer and of eflervescent waters. Seven years ago he was visited by his first attack of gout. Since that time he has had frequent attacks of podagra and gonagra. The last one took place a fortnight before the examination in question. At the left elbow, for some weeks, a tophus had been apparent. The first attacks of oppression were felt while bicycling; later, palpitations and breathlessness were induced by over-exertion. Within the last few months the oppression in the chest increased and was accompanied by radiating pains in the left arm and fingers. The stenocardiac fits were most observable in cold weather, especially during the prevalence of cold winds and fogs, so much so that he was brought to a standstill in the streets. Latterly his medical adviser was able distinctly to note a cardiac dilatation. The urine had a specific gravity of 1015 and showed a trace of albumin but no casts.

In a former paper ${ }^{2} I$ drew attention to the fact that with the help of leaden discs fixed to both the nipples, we are able to ascertain whether the patient has maintained under

1 I avail myself of the method of my late brother, August Schottthat is, a percussion with limitation, by which we are enabled to percuss the anatomical limits of the heart. This is a fact which amongst

Dhers I have often clemonstr.
Maison de Santé at Berlin.

Deutsche Medicinische Wochenschrirt, 1897. examination the same distance from the photographic plate (as well as from the fluorescent screen) to the Roentgen tube. It must be especially noted that the anginal attacks are readily excited by bodily exertion and by mental excitement. A somewhat accelerated pace, the ascent of even a slight incline, even the act of turning round in bed or breathing in cold wind or fog may suffice to cause a stenocardiac fit, acts which, however insignificant they may be, require somewhat increased effort on the part of the heart. Under favourable conditions and especially in a state of absolute rest these accesses generally soon subside. The etiology of the affection is too well known to need special description, but it must not be forgotten that inheritance plays a prominent part in the causation of this disease. I mysel bave observed a good number of cases in which several in dividual members of one family have suffered from angina pectoris.

It now remains to consider the therapy of this disease, and in doing so we shall, I think, discover from further evidence the correctness of the Parry. Stokes theory. Setting aside prophylaxis the treatment may be considered under two heads-viz.: (1) the therapy of the anginal fit itself; and (2) the treatment of the heart in the intervals between attacks. The actual paroxysms are most efficaciously relieved by the nitrites, and on that subject the publications of Sir William Broadbent, Sir T. Lauder Brunton, stc, are so well known that I scarcely need to enter into further details. Nitro-glycerine is in most cases superior to the nitrite of amyl, as its effect is mostly more prompt and intense. I decidedly prefer the liquid form to the tabloids on account of the more rapid effect and the facility of accurate dosage, but we know that inbalations of the nitrite of amyl prove successful in some cases in which nitro glycerine fails to relieve. My experience of the use and value of the tetra. nitrate of erythrol is not sufficiently extensive to justify the expression of a decicted upinion. Nitro-glycerine in suitable cases frequently produces striking effects, but without doubt its application requires much caution and experience and constant medical supervision, as, in default of these safeguards, collapse is only too likely to occur. Antipyrin, formerly much used by the French and especially by Germain Sée, has more and more lost its advocates, not only because of its more or less questionable efficiency, but also on account of its being a source of some danger to the patient. The paroxysm of pain may also be relieved by the application of external measures such as friction with spirit of wine (I prefer hot spirit of wine with an addition of salt) mustard spirit, or the application of mustard leaves, \&c. But most efficacious have I found the application of heat, and for that purpose $I$ have designed a special india-rubber bag provided with a thermometer in such a manner that the temperature of the water may be measured and regulated at any moment. Moving the bag at a temperature of from $140^{\circ}$ to $170^{\circ} \mathrm{F}$. with light tonches over the whole chest effects, in a good number of cases, either marked relief or the absolute suppression of the pains.

In a number of cases, however, we must expect to be disappointed by the above-mentioned remedies and be prepared for the pains to become so intense that we shall be forced to have recourse to narcotics. Before all others stands morphine, but even where it becomes indispensable it is advisable not to bring it into continuous use. It is preferable to give single considerable doses and, if necessary, to administer it subcutaneously. The injurious effect on the heart of the prolonged use of morphine may be regarded as generally admitted. In iodine we have a medicament which is to be used as well during the fits as during their intervals. As all salts of potassium are more or less detrimental to the heart, and as in cases of angina pectoris vera we have to apprehend a failure of power, I always prescribe the iodide of sodium, which in view of the irritating effects of salts of iodine on the mucous membranes, including that of the digestive organs, is best exhibited in milk. In a series of cases it has proved very useful even where no antecedent syphilitic taint existed; in others it was not followed by good effect, but this remedy also ought not to be given for a long-continued period and particularly not if given in increasing doses. Especially will it be necessary carefully to watch the weight of the body, as all the salts of iodine are apt to destroy the molecules of albumin and thereby to cause more injury than any benefit that can accrue.

In the intervals of the attacks and indeed often in the course of the attacks we must take measures to stimulate the heart and to strengthen it. As stimulants we especially 
commend ether or camphor and the salts of caffeine. Among the latter I desire to express a caution as to the use of iodiosalicylate, because I have observed that marked relapse is apt to follow the use of that remedy, whereas the sodiobenzoate and the citrate appear to be exempt from that drawback. It is a well-known fact that digitalis fails to relieve much more frequently in cases of angina pectoris vera than in many other diseases of the heart. This applies as much to the uncomplicated forms caused by the sclerosis of the coronary vessels as to those which are combined with aortic valvular lesions or aortitis. We must not, however, undervalue its usefulness to such an extent as has been done latterly in different directions, for, though the pains be little or not at all influenced by its use, we often succeed in obtaining a beneficial effect from this important drug in cases of heart disease. Still less must we rely upon strophanthus which too often is ineffectual; and still less can we trust to inferior remedies such as convallaria majalis, adonis, and such like remedies which need scarcely be mentioned.

In such cases as we are now considering physical therapy proves its value in a most satisfactory way, and the tonic effect of the balneological and gymnastic treatment as described by my late brother and myself becomes most evident. I abstain from recurring to the details of our system and only lay stress upon the importance of much caution in the application of the baths as well as the therapeutic exercises. I would especially express a warning against hurrying on to strong effervescent baths and especially to the strongest form of the effervescent running baths. Besides, more harm than benefit would be derived from baths either too cool or too warm. Very rarely are we allowed to go below $85^{\circ}$ or to rise above $93^{\circ} \mathrm{F}$., nay, even from a temperature of $95^{\circ} \mathrm{I}$ have now and then observed unfavourable effects. Moreover a long immersion is not advisable. I mostly limit it to the space of 15 minutes and rarely extend it to 20 minutes. Similar precautionary measures are to be observed with regard to exercises. The resistance must not be very strong, the pauses between the exercises must not be too short, and the patient must never be suffered to exert himself till fatigued. A sufficient interval of rest after the exercises is under all circumstances important.

In a series of articles I have shown in past years the results of the balneological and gymnastic treatment especially in cases in which rest as well as pharmaceutic remedies proved useless. Recently their efficacy has been attested by many authorities. In response to inquiries lateiy addressed to me I should be glad to state that the treatment by baths and exercises has proved to be of signal success even in cases in which the nitrites, and principally nitro-glycerine, had more or less failed. I would especially refer to a treatise by Rives. ${ }^{3}$ Rives had the opportunity of observing at Nauheim a patient who was under my treatment and had been sent to me by Sir T. Lauder Brunton. He came from Karachi, was aged 53 years, and was subject to so many stenocardiac fits that in the first few days of his stay at Nauheim he was obliged to have recourse to as many as 20 tabloids of nitro-glycerine a day. A treatment of 13 weeks caused all symptoms not only to disappear but resulted in a general state of well-being. It deserves to be remarked that this patient continued to enjoy good health until great mental strain induced by the ravages of the plague in his town subjected him again to slight attacks. A renewed treatment in Nauheim of seven weeks in the spring and of five weeks in autumn again had the effect of removing his troubles so that Sir T. I sauder Brunton, on the return of the patient, was able to certify a perfectly normal condition of the heart. I must not omit to mention that with such patients it is necessary to adhere to the same precautionary measures as to diet, exercise in fresh air, dress, \&c., as are to be observed with other patients with heart diseases, such as failure of compensation in consequence of valvular lesions, and in myocarditis, \&c.

It must, however, be clearly stated that not all cases without exception are suitable for balneological and gymnastic treatment. When arterio-sclerosis has made such progress that the danger of embolism or apoplexy may be apprehended, or where there is reason to believe that there already exists even a small aneurysm of the wall of the heart or the aorta which might favour rupture, it is well known that every increase of the blood-presspre, which is a natural consequence of the above treatment, might lead to

3 New York Medical Journal, 1896. twe fatal consequences. Rigidity of the vessels or early stages of arterio-sclerosis may be treated by these methods and usually yield satisfactory results, but I must lay stress again upon the fact that advanced sclerosis is a contraindication for this treatment. As regards the latter state I have raised a note of warning years ago, ${ }^{4}$ and my later experience has only strengthened my view on the subject. In such cases strict enforcement of rest is certainly preferable.

Looking, in conclusion, once more at the actual state of our therapeutic experience we are brought to the conclusion that the results which we obtain by the application of the above-mentioned more prominent medicaments is to be explained by the lowering of the pressure of the blood and the moderation of the heart's action consequent upon it. Differing therefrom are the causes which ensure success in the balneological and gymnastic treatment because it is obtained by the exercise of a tonic influence. By strengthening the heart's muscle, as well as by acting on the cardiac nerves, the distressing symptoms of the angina pectoris are either removed or reduced ; in other worde, ex juvantibus, it is shown that the Parry-Stokes theory is based on a real and solid foundation.

Bad-Nauheim.

\section{SOME REMARKS UPON THE TREATMENT OF HEART DISEASE, WITH SPECIAL REFERENCE TO THE "HILL HEART."}

BY H. J. CAMPBELL, M.D., F.R.C.P. LOND.,

SEXIOR PHYSICIAN TO THE BRADFORD ROYAL INFIRMARY AND LECTURER ON FORENSIC MEDICINE IN THE YORKSHIRE COLLEGE, IEEDS.

IN the treatment of most forms of heart disease the difficult problem of how to relieve an organ which has such constant demands made upon it, and which is obliged to rest and recuperate in the brief intervals allowed between its active contractions, is met in one of two ways, the one being the stimulation of the heart, so that by working harder and more efficiently, it may compensate for its own defectiveness, and the other the lessening of the work that the organ has to perform, so that the balance of demand and supply of force may be restored and maintained. In most cases of valvular disease with failing compensation, we of course adopt the two methods of excitation and compensation both at once, combining the exhibition of cardiac stimulants, such as digitalis, strophanthus, convalaria, sparteine. or caffeine, with the diminishing of cardiac work by ordering rest in bed, and the lowering of blood-pressure by the judicious use of purgatives, diuretics, \&c.

In the special methods adopted at Nauheim, the same combination of stimulation with alternate lessening of work, explains the marked success of this form of treatment in suitable cases. The effect of the Sprüdel water at a temperature of $95^{\circ}$ and charged with carbonic acid, is to cause the peripheral vessels to contract and the bloodpressure consequently to rise, as evidenced by the slowing of the pulse. The result is that the heart has more work to do and therefore has its muscle stimulated to more forcible and complete contraction, with the consequent more thorough emptying of its cavities. After leaving the bath the peripheral vessels dilate, the blood.pressure is lowered, and the resulting diminution of the work the heart has to perform, enables the muscle to maintain its increased state of efficiency. Exactly the same sequence of events occurs with the resisted movements, for here again the first effect is to cause a rise of blood-pressure, shown by the slowing of the pulse, while the second effect of dilatation of the vessels of the muscle lowers blood-pressure and therefore lessens the strain upon the labouring heart. In this respect it is interesting to note that Dr. Schott has demonstrated by the use of the Roentgen rays that the more complete emptying of the heart and the consequent reduction of dilatation under this treatment, is an actual fact and is

4 The Lancet, May 23rd, p. 1143, and May 30th, 1891, p. 1199. 1 A paper read before the Yorkshire Branch of the British Medical Association on March 28th, 1900, at Huddersfield. 